Larkina S. A., Seletskaya A. V., Makarenko O. A., Vastyanov R. S. Rat skin inflammation markers change in response to calcium hydroxyapatite and hyaluronic acid administration. Journal of Education, Health and Sport. 2021;11(08):510-519. eISSN 2391-8306. DOI http://dx.doi.org/10.12775/JEHS.2021.11.08.057

https://apcz.umk.pl/JEHS/article/view/JEHS.2021.11.08.057

https://zenodo.org/record/5579678

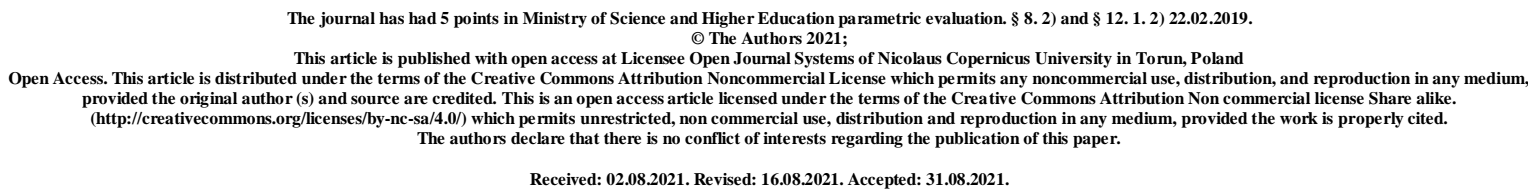

UDK 65.012.1+599.323.4:616.314.17-008.1

\title{
RAT SKIN INFLAMMATION MARKERS CHANGE IN RESPONSE TO CALCIUM HYDROXYAPATITE AND HYALURONIC ACID ADMINISTRATION
}

\author{
S. A. Larkina ${ }^{1}$, A. V. Seletskaya ${ }^{2}$, O. A. Makarenko³ ${ }^{3}$ R. S. Vastyanov ${ }^{1}$ \\ ${ }^{1}$ Odessa National Medical University, Odessa, Ukraine \\ ${ }^{2}$ Kings College, London, UK \\ ${ }^{3}$ I. I. Mechnikov National University, Odessa, Ukraine
}

\begin{abstract}
In the research, the level of some markers of skin inflammation in Wistar rats was examined after injection of calcium hydroxyapatite and hyaluronic acid drugs of various concentrations, where the nature, degree, extent of tissue damage and the general condition of the animals in general were close to humans'. Each drug was evaluated according to several indicators (MDA content, activity of neutrophil elastase, acid phosphatase, and catalase) to understand the measure of the adequacy of the body's response to the intensity of the stimulus.

A biochemical analysis of the skin of laboratory rats after the injection of the studied drugs showed that the safest drugs contain nonstabilized $4 \%$ hyaluronic acid. Either in pure form or in a 1: 1 mixture with calcium hydroxyapatite at a concentration of $55.7 \%$, since in 2 months after their injection in the skin of experimental animals, markers of inflammation (elastase, acid phosphatase and MDA levels), catalase and API activity were found at the normal level.
\end{abstract}

The injection of drugs containing stabilized $2 \%$ hyaluronic acid and calcium hydroxyapatite $55.7 \%$, as well as stabilized $2.6 \%$ hyaluronic acid and calcium hydroxyapatite 
at a concentration of $1 \%$ caused prolonged LPO activation, increased level of inflammation markers and decreased API.

The injection of calcium hydroxyapatite drugs without hyaluronic acid, even at a 1: 1 dilution with saline, also caused prolonged LPO activation, an increased level of inflammation markers and a decreased API, and, in addition, revealed a significant increase in acid phosphatase activity, which is an indicator of the integrity of cell membranes.

The obtained results made it possible to analyze the features of the molecular response and regulation of inflammation upon injection of calcium hydroxyapatite and hyaluronic acid drugs of different concentrations, which will help in prescribing of treatment.

Key words: calcium hydroxyapatite; hyaluronic acid; skin; markers of skin inflammation; skin cell membrane factors; connective tissue; collagen; involutive skin changes; skin aging; anty-age.

Introduction. In the modern world, the development of methods of prevention and treatment of age-related changes in the skin and activation of regenerative processes is relevant and has great scientific and practical significance, but due to the specificity of the molecular response, the principles of control and regulation of inflammation processes have not been fully studied. The number of complications after the introduction of various drugs into human skin increases [1,2].

The course of inflammation depends on local factors (composition of the drug, nature, degree and extent of tissue damage, etc.) and general (initial state of the body, immune, neuro-endocrine status). Inflammation can have a positive effect by increasing the synthesis of prostaglandins, leukotrienes, and renewal of phospholipid membrane structures. Depending on the goals of therapy and the initial data, the inflammatory process must be stimulated in some cases and suppressed in others [3].

Aim of the work: To study the indicators of markers of skin inflammation after injection of calcium hydroxyapatite and hyaluronic acid drugs of various concentrations, which are widely used in medicine.

\section{Materials and methods}

The study was carried out on 92 Wistar rats of herd breeding, females aged 6-7 months with an average weight of $285 \pm 34 \mathrm{~g}$. The animals were divided into 6 groups depending on the drug used, which was injected subcutaneously in the volume of $1.0 \mathrm{ml}$.

1. Control group - $\mathrm{NaCl} 0.9 \%$ 
2. Calcium hydroxyapatite $55.7 \%$ with hyaluronic acid $2 \%$ (HArmonyCA, LUMINERA)

3. Calcium hydroxyapatite $55.7 \%$ diluted 1: 1 with $0.9 \% \mathrm{NaCl}$ (Radiesse, Merz)

4. Calcium hydroxyapatite $55.7 \%$ with hyaluronic acid 4\% (Crystalys diluted 1: 1 with Hydrial 4\%, LUMINERA)

5. Hyaluronic acid 4\% (Hydrial 4\%, LUMINERA)

6. Calcium hydroxyapatite $1 \%$ with hyaluronic acid $2.6 \%$ (Neauvia stimulate).

The drugs were injected into the back, $1 \mathrm{~cm}$ lateral to the spine, using a syringe with a solution, linear retrograde with a $27 \mathrm{G}$ needle. When working with animals, we were guided by the Law of Ukraine "On the Protection of Animals from Cruelty" (No. 1759-VI of December 15, 2009), taking into account the rules of the European Convention "On the Protection of Vertebrate Animals Used for Experimental and Other Scientific Purposes".

The animals were removed from the experiment 60 days after the injection of drugs under thiopental anaesthesia $(40 \mathrm{mg} / \mathrm{kg})$. Pre-shaved skin areas of 3.0 by $1.5 \mathrm{~cm}^{2}$ were isolated in the area of drug injection. The skin was frozen prior to analysis.

Skin homogenates were prepared at the rate of $75 \mathrm{mg} / \mathrm{ml} 0.05 \mathrm{M}$ Tris- $\mathrm{HCl}$ buffer, $\mathrm{pH}=7.5$, and the supernatant obtained after centrifugation at $2500 \mathrm{rpm}$ for $30 \mathrm{~min}$ was used for biochemical studies.

To assess the degree of inflammation in the injection sites of the skin of rats, after the injection of the studied drugs, the markers of inflammation were determined: the content of malondialdehyde (MDA), the activity of neutrophilic elastase, acid phosphatase and catalase.

The acid phosphatase activity ( $\mathrm{pH} 4.8)$ was determined by the method of Bessey at al. on the hydrolysis of n-nitrophenyl phosphate ("Sigma", Germany).

The elastase activity was assessed by the Visser and Blout method by the hydrolysis of the substrate N-t-BOC-L-alanin-p-nitrophenyl ester (Sigma, Germany).

The content of malonic dialdehyde (MDA) was determined by reaction with 2thiobarbituric acid [4].

Catalase activity in rat skin was determined using ammonium molybdate [5].

The antioxidant-prooxidant index (API) was calculated from the ratio of catalase activity to MDA content [6].

The static processing of the obtained results was carried out according to the generally accepted Student-Fisher method [7]. 


\section{Results}

The results of the biochemical analysis of the skin of rats after injections of the used drugs are summarized in Table 1 and are presented in the figures.

Table 1

The content of MDA, the activity of neutrophilic elastase, acid phosphatase and catalase in the skin of rats after 2 months after the use investigated drugs administration

\begin{tabular}{|l|c|c|c|c|}
\hline $\begin{array}{c}\text { № } \\
\text { of the } \\
\text { group }\end{array}$ & $\begin{array}{c}\text { MDA content, } \\
\text { mmol/kg }\end{array}$ & $\begin{array}{c}\text { Acid phosphatase } \\
\text { activity, } \\
\text { mk-cat/kg }\end{array}$ & $\begin{array}{c}\text { Elastase activity } \\
\text { mk-cat/kg }\end{array}$ & $\begin{array}{c}\text { Catalase activity, } \\
\mu \text { cat/kg }\end{array}$ \\
\hline 1. & $1,90 \pm 0,10$ & $12,69 \pm 1,0$ & $12,00 \pm 0,84$ & $2,40 \pm 0,14$ \\
\hline 2. & $3,01 \pm 0,12$ & $15,34 \pm 1,0$ & $16,18 \pm 1,13$ & $2,26 \pm 0,10$ \\
& $\mathrm{p}<0,002$ & $0,05<\mathrm{p} 1<0,1$ & $\mathrm{p}<0,01$ & $\mathrm{p}>0,4$ \\
\hline 3. & $3,10 \pm 0,24$ & $19,34 \pm 1,33$ & $11,84 \pm 1,56$ & $2,94 \pm 0,13$ \\
& $\mathrm{p}<0,001$ & $\mathrm{p}<0,002$ & $\mathrm{p}>0,9$ & $\mathrm{p}<0,01$ \\
\hline 4. & $2,09 \pm 0,15$ & $11,09 \pm 0,86$ & $12,16 \pm 0,97$ & $2,83 \pm 0,14$ \\
& $\mathrm{p}>0,3$ & $\mathrm{p}>0,25$ & $\mathrm{p}>0,8$ & $0,05<\mathrm{p} 1<0,1$ \\
\hline 5. & $2,18 \pm 0,13$ & $11,61 \pm 0,96$ & $13,01 \pm 0,97$ & $2,33 \pm 0,15$ \\
& $\mathrm{p}>0,1$ & $\mathrm{p}>0,5$ & $\mathrm{p}>0,4$ & $\mathrm{p}>0,7$ \\
\hline 6. & $3,34 \pm 0,21$ & $13,6 \pm 0,78$ & $15,41 \pm 1,25$ & $2,56 \pm 0,13$ \\
& $\mathrm{p}<0,001$ & $\mathrm{p}>0,5$ & $\mathrm{p}<0,02$ & $\mathrm{p}>0,4$ \\
\hline
\end{tabular}

Note: $p$ - reliability of differences to the indicator in group No. 1 (control group physical solution)

Fig. 1 shows the data of the determination of the level of malondialdehyde (MDA). This indicator is a secondary product of lipid peroxidation (LPO), and therefore it is considered to be a marker of LPO, the intensity of which, as you know, increases in almost any pathology and inflammation, in particular.

As shown in Fig. 1 injection of all drugs led to an increase in the MDA content in the skin of rats. Thus, the level of this LPO marker exceeded the values in the skin of rats of the $1^{\text {st }}$ group (control-saline solution) by $58.4 \%$ compared to the skin of rats in the 2nd group ( $\mathrm{p}<0.002)$, by $63.2 \%$ - in the skin of rats of the 3rd group ( $<<0.001)$, unreliable by $10.0 \%$ - in the skin of rats of the 4th group ( $>>0.3$ ), unreliable by $14.7 \%$ - in the skin of rats of the 5 th group ( $>>0.1)$ and by $75.8 \%$ - in the skin of rats of the 6th group $(p<0.001)$.

Comparing the obtained results, we can distinguish the highest intensity of LPO in the skin of rats after the injection of drugs from groups No. 3 and No. 6, a lower level of MDA, 
which is not significantly distinguishable from the values of the 1st group, was registered after the use of drugs from groups No. 4 and No. 5.

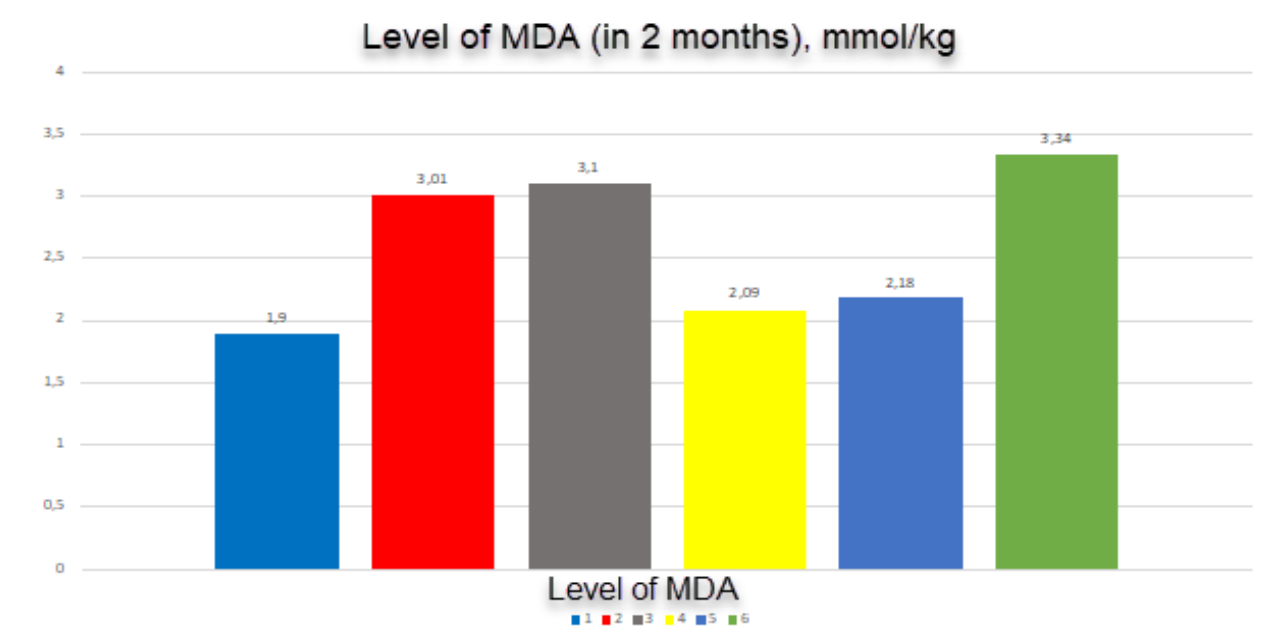

Fig. 1. The level of malondialdehyde (MDA) in the skin of rats 2 months after injection of drugs, $\mathrm{mmol} / \mathrm{kg}$

Apparently, the high intensity of lipid peroxidation in the skin of animals even in 2 months after the injection of drugs is associated with the features of skin reactivity to the effect of drugs. It is known that LPO is not only a pathological factor, but also physiologically necessary for the synthesis of prostaglandins, leukotrienes, processes of phagocytosis, pinocytosis, as well as an integral mechanism for the renewal of phospholipid membrane structures.

LPO is under the control of a multicomponent physiological antioxidant system, one of which is the antioxidant enzyme catalase. The data on the determination of the activity of this enzyme in the skin of animals are summarized in Fig. 2, which shows that significant differences in the increase in catalase activity, registered only in the skin of rats, which were injected with drug No. 3 , by $22.5 \%(\mathrm{p}<0.01)$. 


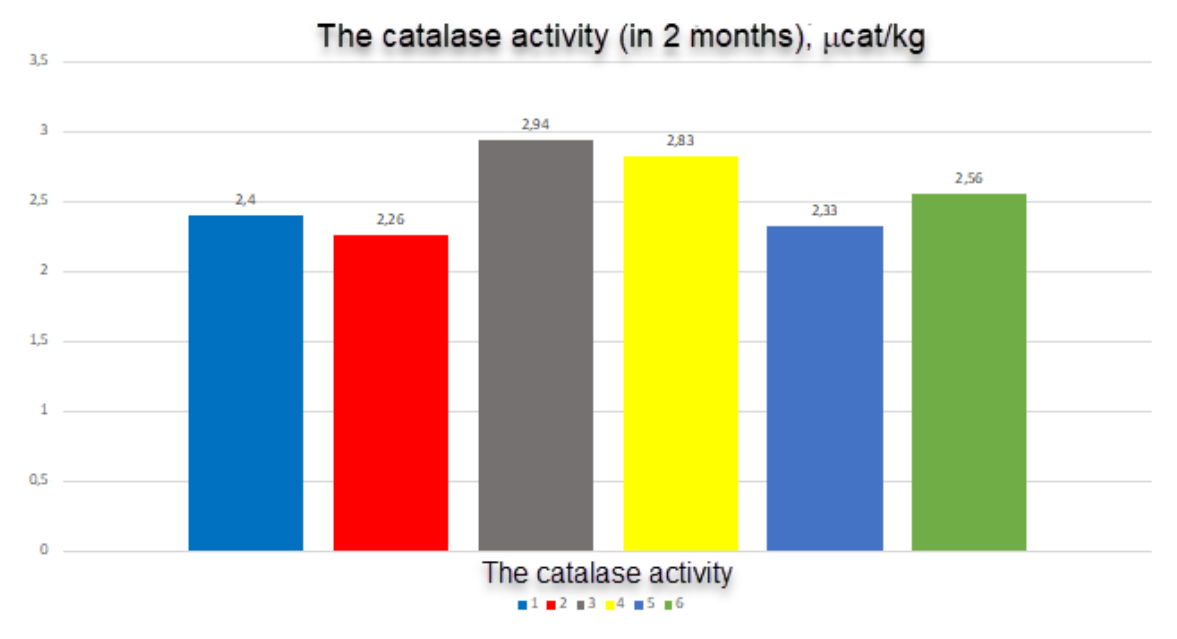

Fig. 2. Catalase activity in rat skin 2 months after drug injection, $\mu$ cat $/ \mathrm{kg}$

Catalase activity in rat skin after injection of drug No. 4 had increased by $17.9 \%$ $(0.05<\mathrm{p} 1<0.1)$. In the skin of animals of other groups, the activity of this antioxidant enzyme did not differ from the level in the skin of rats of the 1st group, which was injected with saline (p> 0.1-0.7, Fig. 2).

The ratio of antioxidant components to the prooxidant components, or rather the predominance of peroxidation activation or its suppression due to the high activity of antioxidants, reflects the API index. The results of calculation of this indicator are shown in table. 2

Table 2

Results of the API index in he rats' skin 2 months after drug injection

\begin{tabular}{|c|l|}
\hline $\begin{array}{c}\text { № } \\
\text { of the group }\end{array}$ & \multicolumn{1}{|c|}{ API antioxidant-prooxidant index } \\
\hline 1 & $12,63 \pm 0,96$ \\
\hline 2 & $7,51 \pm 0,52 \mathrm{p}<0,001$ \\
\hline 3 & $9,76 \pm 0,940,05<\mathrm{p} 1<0,1$ \\
\hline 4 & $13,54 \pm 1,04 \mathrm{p}>0,5$ \\
\hline 5 & $10,68 \pm 0,82 \mathrm{p}>0,2$ \\
\hline 6 & $7,66 \pm 0,51 \quad \mathrm{p}<0,001$ \\
\hline
\end{tabular}

A significant decrease in the level of API by an average of $40.0 \%$ was observed in the skin of rats, which were injected with drugs from groups No. 2 and No. $6(p<0.001)$. This was the result of a high MDA content and low catalase activity (inability of the antioxidant defense to suppress lipid peroxidation) and indicates the depletion of antioxidant protection and the predominance of lipid peroxidation in the skin of these animals. 
The tense state of the antioxidant system can be concluded by the API in the skin of rats of the 3rd group after the injection of the drug of the group No. 3. In this group, the level of MDA was significant (Fig. 1), but the highest activity of catalase, and, therefore, antioxidant protection (Fig. 2) is capable of inhibiting excessive lipid peroxidation processes. And the values of the API indicator, which decreased $(0.05<\mathrm{p} 1<0.1)$, became a confirmation of this (Table 2).

In the skin of animals that were injected with drugs from groups No. 4 and No. 5, API was high at the level of values in control rats $(p>0.2-0.5)$. These data indicate a high antioxidant activity of drugs from group No. 4 and No. 5, capable of effective suppression of lipid peroxidation (Table 2).

The results of determining the activity of acid phosphatase are shown in Fig. 3. It is an indicator of membrane integrity, since it is located in lysosomes and its activity increases with increasing membrane permeability

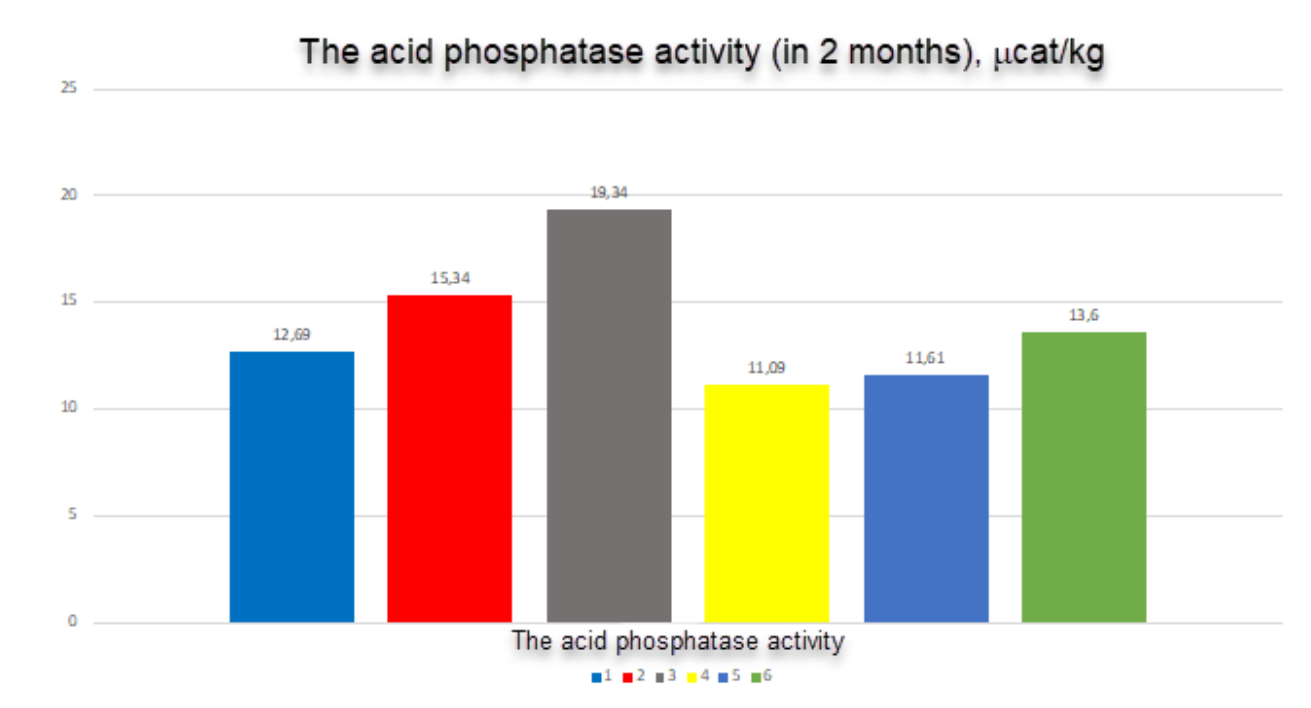

Fig. 3. Activity of acid phosphatase in the skin of rats 2 months after injection of drugs, $\mu \mathrm{cat} / \mathrm{kg}$.

The analysis revealed a significant increase in the activity of this inflammation marker only in the skin of group 3 rats by $52.4 \%(p<0.002)$. In the skin of rats of the 2 nd group after injection, the activity of acid phosphatase tended to increase - by $20.9 \%(0.05<\mathrm{p} 1<0.1)$.

In the remaining groups, a constant level of this indicator was recorded in relation to the values in the skin of rats, which were injected with saline $(p>0.25-0.5)$. The above results indicate the absence of a damaging effect on the membranes of skin cells of experimental animal drugs from groups 4, 5 and 6 (Fig. 3). 
It is known that the degree of elastase activity in tissues reflects the intensity of destruction of elastic fibers. The source of this destructive enzyme is segmented neutrophils. The results of determining the activity of elastase in rat skin are shown in Fig. 4.

The conducted studies revealed a significantly increased level of this marker of inflammation after injection of drugs in group No. 2 - by $34.8 \%(\mathrm{p}<0.01)$ and No. 6 - by $28.4 \%(\mathrm{p}<0.02)$. The use of drugs in groups No. 3, No. 4 and No. 5 did not have a significant effect on the activity of elastase in the skin of animals ( $p>0.4-0.9$ ), which indicates the absence of an inflammatory reaction (Fig. 4).

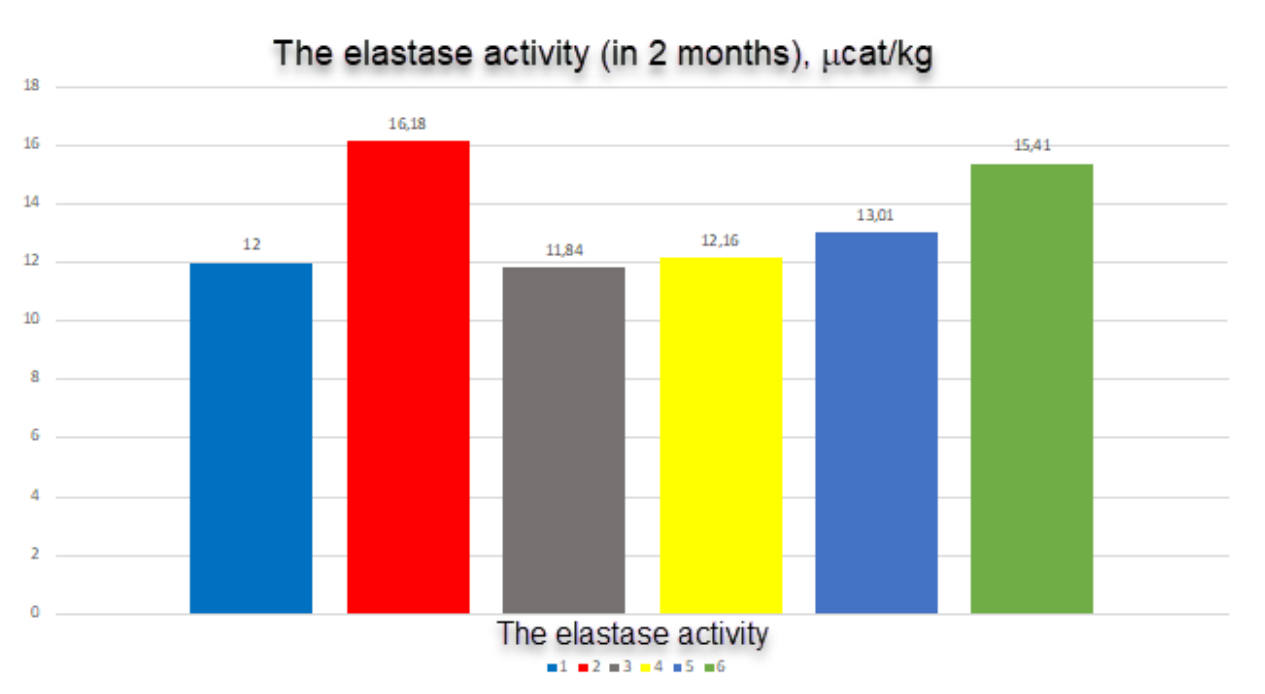

Fig. 4. Activity of elastase in the skin of rats 2 months after injection of drugs, $\mu-\mathrm{cat} / \mathrm{kg}$

\section{Discussion}

The biochemical analysis of the skin of laboratory rats after the injection of the investigated drugs allows to confirm the pronounced anti-inflammatory activity of drugs of nonstabilized hyaluronic acid at a concentration of $4 \%$, both in pure form and in a mixture with calcium hydroxyapatite [8]. This allows us to recommend them to patients with risks of post-inflammatory complications.

The injection of drugs containing stabilized hyaluronic acid and calcium hydroxyapatite, regardless of concentration, caused prolonged LPO activation against the background of an increased level of inflammation markers and a decreased API. Perhaps such drugs are more suitable for young and healthy patients without a burdened history, since 
inflammation can have a positive effect in terms of increasing the synthesis of prostaglandins, leukotrienes, and renewal of phospholipid membrane structures.

Injection of calcium hydroxyapatite drugs without hyaluronic acid caused prolonged LPO activation and increased cell membrane permeability.

\section{Conclusions}

1) Drugs containing nonstabilized $4 \%$ hyaluronic acid in pure form, in 2 months after their injection in the skin of experimental animals showed that markers of inflammation (elastase, acid phosphatase and MDA levels), catalase and API activity are at the normal level.

2) Drugs containing nonstabilized $4 \%$ hyaluronic acid in a 1:1 mixture with calcium hydroxyapatite at a concentration of $55.7 \% 2$ months after their injection in the skin of experimental animals showed that inflammation markers (elastase activity, acid phosphatase and MDA level), the activity of catalase and API are at the normal level.

3) The injection of drugs containing stabilized $2 \%$ hyaluronic acid and calcium hydroxyapatite $55.7 \%$ caused prolonged LPO activation and an increased level of inflammation markers and a reduced API by $40.0 \%$.

4) Injection of drugs containing stabilized $2.6 \%$ hyaluronic acid and calcium hydroxyapatite at a concentration of $1 \%$ caused prolonged LPO activation and an increased level of inflammation markers and a $39.0 \%$ decrease in API.

5) The injection of calcium hydroxyapatite drugs without hyaluronic acid, even at a 1:1 dilution with saline, caused prolonged LPO activation, an increased level of inflammation markers and a decreased API, and, in addition, revealed a significant increase in acid phosphatase activity by $52.4 \%$ which is an indicator of the integrity of cell membranes and its activity increases with increasing membrane permeability.

\section{References}

1. Tatuzyan E.G., Belovol A.N., Tkachenko S.G. Complications of minimally invasive procedures in cosmetology. Dermatology and Venereology. 2015; 2 (64): 79-84 [In Russian].

2. Karpova E.I., Potekaev N.N., Murakov S.V., Danishchuk OI, Demina O.M. Complications of contouring injection plastic of the face: world and Russian experience. Plastic surgery and aesthetic medicine. 2019; 4: 54-75 [In Russian].

3. Wismont F.I. Inflammation. Pathophysiological aspects. Minsk, 2006: 48 [In Russian]. 
4. Stalnaya I.D, Garishvili T.G. Modern methods in biochemistry. M : Medicine, 1977: 66 - 68. [In Russian].

5. Karolyuk M.A., Ivanova L.I., Mayorova N.T., Tokarev K.E. Method for determining the activity of catalase. Laboratory work. 1988; 1: 16-18. [In Russian].

6. Levitsky A.P, Pochtar V.M., Makarenko O.A., Gridina L.I. Antioxidantprooxidant index of blood serum of rats with experimental stomatitis and its correction with dental elixirs. Odessa Medical Journal. 2006; 1 (93): 22-25 [In Ukrainian].

7. Lapach S.N., Chubenko A.V., Babich P.N. Statistical methods in biomedical research using Excel. K : Morion, 2000: 320 [In Russian].

8. Larkina S., Makarenko O., Seletskaya A. Study of the anti-inflammatory activity of hyaluronic acid preparations. News of aesthetic medicine. 2019; 6 (118): 58-63 [In Russian]. 